\title{
Intron-mediated enhancement is not limited to introns
}

\author{
Jenna E Gallegos \& Alan B Rose
}

\begin{abstract}
Certain introns strongly increase mRNA accumulation by a poorly understood mechanism known as Intron-Mediated Enhancement (IME). Introns that boost expression by IME have no effect when located upstream of or more than $\sim 1 \mathrm{~Kb}$ downstream from the start of transcription. The sequence TTNGATYTG, which is over-represented in promoter-proximal introns in Arabidopsis thaliana, can convert a non-stimulating intron into one that strongly increases mRNA accumulation. We tested the ability of an intron containing this motif to stimulate expression from different locations and found that it had the same positional requirements as naturally occurring IME introns. The motif also stimulated gene expression from within the 5'-UTR and coding sequences of an intronless construct. Furthermore, the 5'-UTR of another gene increased expression when inserted into an otherwise non-stimulating intron in coding sequences. These results demonstrate that splicing is not required for intron-mediated enhancement, and suggest that other sequences downstream of the transcription start site in addition to introns may stimulate expression by a similar mechanism.
\end{abstract}

\section{Introduction}

Significant efforts have been invested in identifying the DNA sequences that control the expression of individual genes in eukaryotes. These studies have revealed many common kinds of regulatory elements that collectively constitute promoters in the broadest sense of the term. These include the sites surrounding and immediately upstream of the transcription start sites (TSSs) to which general transcription factors bind to form the pre-initiation complex, proximal binding sites (usually less than $1 \mathrm{~kb}$ from the TSS) for regulatory transcription factors, and distal elements, such as enhancers, which can affect expression over great distances in either direction (reviewed in [1-5]).

In addition to these well-known regulators of transcription, other transcribed sequences can play an important role in controlling expression. 5' and 3' UTR's have been shown to influence mRNA stability, export, and translation (reviewed in [6-8]), exons can contain transcription factor binding sites [9] or intragenic enhancers [10], and introns have been shown to affect gene expression by a number of known and unknown mechanisms.

Some introns contain enhancers [11,12], alternative transcription start sites [13], or transcription factor binding sites [14]. In addition, splicing can have a general positive effect on expression via coupling with other mRNA processing events such 
as capping and polyadenylation [15]. Deposition of the exon junction complex proteins also aids in mRNA export and translation [16,17], and splicing can influence transcription by affecting the phosphorylation state of RNA polymerase II [18].

Certain introns can increase gene expression by an additional, poorly understood mechanism referred to as intron mediated enhancement (IME) [19]. Several properties of IME indicate that these introns influence expression in a manner that is mechanistically distinct from enhancers or proximal promoter elements. The most thoroughly analyzed IME intron is the first intron from the Arabidopsis UBQ10 gene. This intron increases mRNA accumulation only when located downstream of the TSS, and can stimulate expression from at least $550 \mathrm{nt}$ from the TSS but not $1,100 \mathrm{nt}$ or more[20,21]. Remarkably, deleting a $300 \mathrm{nt}$ region of the proximal promoter that contains all known TSSs does not diminish the expression of constructs containing this intron [21]. Transcription in these promoter-deleted constructs initiates in normally untranscribed sequences the same distance upstream of the intron as when the promoter is intact. The observation that many introns stimulate gene expression only from within transcribed sequences near the 5 '-end of a gene [20,22-24] is the basis for the IMEter algorithm described below.

The role of intron splicing in IME is a subject of some debate [19]. Splicing is clearly not sufficient for IME, because many efficiently spliced introns have no effect on expression [25]. Testing whether or not splicing is necessary for IME is complicated by the fact that disrupting splicing has many consequences. Constructs with an unspliceable intron produce mRNA that differs in size and structure from constructs containing a spliceable intron or an intronless control. Thus mRNA with a retained intron may differ in stability or translatability. The intron sequences retained in the mRNA can also cause frame shifts or contain premature start or stop codons, all of which might abolish translation of the reporter gene and lead to mRNA instability through nonsense-mediated mRNA decay. In cases where splicing was prevented but the reading frame was preserved by adjusting intron length and eliminating inframe start and stop codons, expression levels were reduced but not eliminated [25-28]. The degree to which expression levels dropped varied greatly by species and the size, location, and original stimulating ability of the intron, precluding broad conclusions about the need for splicing in IME.

The differing ability of spliced introns to increase mRNA accumulation implies that some must contain stimulating sequences that others lack. These sequences have proven difficult to identify because they are redundant and dispersed throughout stimulating introns [26,29]. Progress was made using the IMEter algorithm, which generates a score that reflects the degree to which the oligomer composition of a given intron resembles that of promoter-proximal introns genome-wide. High IMEter scores have accurately predicted the stimulating ability of introns in Arabidopsis [29], soybeans [30], and other angiosperms [31]. The IMEter does not directly reveal stimulating sequences but can be used to identify sufficient numbers of potentially stimulating introns to allow computational searches for shared sequences. 
One such motif, TTNGATYTG, was found to be over-represented in introns with high IMEter scores [29]. Rearranging nucleotides to create 6 or 11 copies of this motif converted a non-stimulating intron from the COR15a gene in Arabidopsis into one that boosts mRNA accumulation 14- or 24-fold, respectively [32]. Introns containing this motif behave similarly to the $U B Q 10$ intron in that they increase mRNA accumulation even in the absence of the proximal promoter [21], suggesting that the sequence TTNGATYTG is sufficient for IME.

The identification of the TTNGATYTG motif provided an opportunity to determine whether the mechanism of IME requires splicing, and is therefore specific to introns, or if it could act to increase expression from any location within the first few hundred nucleotides of transcribed sequences. We therefore tested the ability of this sequence to stimulate gene expression from within promoter-proximal exon sequences of an intronless construct. We found that six copies of the motif in exon sequences stimulate mRNA accumulation almost as much as does an intron containing six copies of the motif. This demonstrates that splicing is not required for IME, and that the sequences that increase expression by an IME mechanism can also function in exons near the start of a gene.

\section{Results}

The TTNGATYTG motif stimulates expression over a limited range. To first determine if the TTNGATYTG motif has positional requirements similar to the $U B Q 10$ and other natural introns, the ability of an intron engineered to contain 11 copies of the motif to stimulate expression was tested from five locations within a TRP1:GUS reporter construct. This intron, which was previously generated and designated COR15a11L [32], was created by rearranging sequences within a naturally non-stimulating intron from the COR15a gene [25,29]. The COR15a11L intron was inserted into TRPI:GUS fusion constructs at one of five locations: upstream of the transcription start site, within the 5' UTR, at the 3' end of sequences derived from TRP1 exon 1, near the middle of the GUS gene, or at the 3' end of the GUS gene (Figure 1A).

TRPI:GUS constructs containing the COR15a11L intron at the different locations were transformed into Arabidopsis and expression levels were compared. GUS activity was determined by histochemical staining of kanamycin-resistant $\mathrm{T}_{2}$ seedlings in lines of unknown copy number but whose segregation ratios indicated a single locus of insertion. The COR15a11L intron strongly stimulated expression from the 5' end of the gene [32], but had no effect from upstream of the TSS and little effect from the 3 ' end of the gene (Figure 1B).

To determine the level of expression responsible for the observed difference in GUS activity, RNA gel blots were performed on single-copy transgenic lines in which the intron was inserted near the TSS. The differences in GUS activity of these lines is reflected in the steady state GUS mRNA levels, indicating that the intron must be downstream of the TSS to increase mRNA accumulation (Figure 1C). The lack of 
stimulating effect of the COR15a11L intron from the 3' end of the TRP1:GUS fusion or upstream of the TSS rules out the possibility that the TTNGATYTG motif acts as a conventional enhancer or transcription factor binding-site, and suggests that it increases mRNA accumulation by an IME mechanism.

The TTNGATYTG motif stimulates expression from within exons in the absence of splicing. To determine if sequences involved in IME can stimulate expression in the absence of splicing, six copies of the sequence TTAGATCTG (the most active tested version of the TTNGATYTG motif [32]) were engineered into the first $450 \mathrm{nt}$ of transcribed sequences of an intronless TRP1:GUS fusion (Figure 2). The TRP1 sequences are described as exons 1, 2, or 3 based on their location in the endogenous TRP1 gene. Five motifs were introduced into TRP1 exon 1 sequences, two of which were in the 5' UTR, and one copy was introduced into TRP1 exon 2 sequences (Figure 3A). As a negative control, a second TRP1:GUS fusion was generated in which the AT dinucleotide at the center of each motif was changed to TA, making the motif TTAGTACTG. This small inversion was previously shown to eliminate virtually all of the motif's effect on mRNA accumulation from within an intron [32].

Locations for introducing the motifs were selected to minimize changes to mRNA and protein structure (Figure 2). Existing sequences were searched for nine contiguous nucleotides composed of two As, one C, two Gs, and four Ts. Sequences that matched this criterion, or differed by no more than two nucleotides, were rearranged into the sequence TTAGATCTG or TTAGTACTG. In this way, the mRNAs from the tested constructs and controls would remain virtually unchanged in GC content and length. Locations were also selected to maximize the degree to which the changes in amino acids were conservative and consistent with the composition of chloroplast transit peptides. The first two exons of the TRP1 gene encode a chloroplast transit peptide [33], which are rich in serines and threonines and usually devoid of negatively charged amino acids [34]. Chloroplast transit peptides are also poorly conserved and are cleaved off during chloroplast import [35]. Therefore, the changes made to introduce motifs were expected to have minimal effects on the activity of the mature GUS protein.

The stimulating ability of the motif in exonic locations was compared with that of the COR15a6L intron located between TRP1 exon 1 and exon 2 sequences. The COR15a6L intron was previously generated by introducing six copies of the TTNGATYTG motif into the non-stimulating COR15a intron [32]. Expression was measured in single-copy transgenic Arabidopsis at both the mRNA and enzyme activity level and compared to intronless TRP1:GUS controls (Figure 3, Supplemental Tables 1 \& 2).

The intronless TRP1:GUS fusion containing six copies of the TTAGATCTG motif in exons showed substantially more histochemical staining for GUS activity than either the unmodified intronless control or the construct containing the mutated motif (Figure 3B, Supplemental Table 2). Single copy lines containing TRP1:GUS fusions with the TTAGATCTG motif in exons accumulated on average 7.4 times more 
TRP1:GUS mRNA than did the unmodified intronless control (Figure 3C, Supplemental Table 1). This is slightly less than the 9.3 fold increase in expression caused by six copies of the same motif within the COR15a6L intron. In contrast, the fusion containing the mutated motif produced about the same amount of mRNA as the unmodified intronless control. Therefore, the increase in mRNA accumulation caused by the motif is similar in intronic and exonic locations, indicating that the TTAGATCTG motif can boost expression in the absence of splicing.

5'-UTR sequences can stimulate expression from within an intron. The ability of intron sequences to affect expression from the 5'-UTR, and the observation that average IMEter scores are nearly as high for 5'-UTRs as they are for promoterproximal introns, supports the idea that sequences other than introns might boost expression by an IME mechanism [36]. To test this idea, we identified a 5' UTR with a high IMEter score and examined the ability of this sequence to increase expression when inserted into a non-stimulating intron in a TRP1:GUS fusion.

The 5'-UTR chosen was a $74 \mathrm{nt}$ fragment from the $\mathrm{At5g} 53000$ gene, which encodes the Tap 46 regulatory subunit of protein phosphatase $2 \mathrm{~A}$ [37]. This fragment was used to replace an $80 \mathrm{nt}$ internal portion of the COR15a intron, which was located at the 3' end of TRP1 endogenous first exon of a TRP1:GUS fusion.

The expression of each construct and an unmodified intronless control was measured as TRP1:GUS mRNA and GUS enzyme activity in single-copy transgenic Arabidopsis. The At5g53000 5'-UTR fragment stimulated mRNA accumulation more than 2-fold from within the previously non-stimulating COR15a intron (Figure 4, Supplemental Table 1). The effect of the 5'-UTR fragment on GUS enzyme activity (5.7-fold) was roughly twice that seen at the level of mRNA accumulation, as has been observed previously for introns in TRP1:GUS constructs $(20,25,32)$. Therefore, the high IMEter score of this 5'-UTR fragment is biologically relevant. The ability of this non-intron sequence to stimulate expression from within an intron further supports the idea that sequences that increase expression by an IME mechanism may not be limited to introns.

\section{Discussion}

Sequences associated with IME can contribute to mRNA accumulation in a position dependent and splicing independent manner. Multiple copies of the TTNGATYTG motif, which is overrepresented in introns with high IMEter scores, stimulate gene expression more than ten-fold from within a normally nonstimulating intron [32]. Here we showed that introns containing this motif strongly stimulated expression only from within transcribed sequences near the 5' end of a gene. This locational specificity is a hallmark of introns that exhibit IME [20,21], further demonstrating that the TTNGATYTG motif is sufficient for IME. Because some introns that are known to stimulate expression in a position-dependent manner contain few matches to this motif, there must also be other sequences that act similarly. 
Two lines of evidence suggest that IME is predominantly splicing-independent. First, the degree to which mRNA accumulation was increased by six copies of the TTNGATYTG motif was similar when the motifs were located within exons or in an intron. Second, IMEter scores, which strongly correlate with the ability of an intron to increase mRNA, are generally high in 5'-UTRs and to a lesser degree coding sequences near the start of a gene [36]. The ability of a high-scoring 5'UTR fragment from the At5g53000 gene to stimulate mRNA accumulation from within the previously non-stimulating COR15a intron suggests that high IMEter scores of promoter-proximal non-intron sequences might also reflect their potential to increase mRNA accumulation by the same mechanism.

Even though the TTAGATCTG motifs increased mRNA accumulation from locations outside of introns, the effect of those sequences was somewhat higher when they were located within an intron. Thus, while the ability of intron sequences to stimulate gene expression is predominantly splicing independent, splicing may also contribute to an increase in mRNA accumulation. The effect of splicing on mRNA levels could be due to known interactions between the splicing and transcription machinery that increase transcription initiation or elongation, or synergies between splicing and other steps of mRNA processing. The overall level of expression is likely determined by multiple mechanisms, some of which are splicing-dependent and some of which are sequence-dependent.

The observations that intron sequences can stimulate gene expression from coding sequences outside of the context of an intron, that a 5'-UTR sequence with a high IMEter score can increase mRNA accumulation from within an intron, and that average IMEter scores are high genome-wide in 5'-UTRs, suggests that the mechanism through which expression is stimulated might include other sequences near the 5' end of genes. The IMEter may thus be a useful tool for identifying potentially stimulating sequences in both exons and introns.

Possible mechanisms. The properties of the described phenomenon are difficult to reconcile with known mechanisms of gene regulation. Many of the proposed mechanisms for IME such as gene looping or antisense suppression are predicted to require splicing [38,39], while the specific phenomenon reported here, which relates to high IMEter scores, appears to be predominantly splicing-independent.

It is unlikely that mRNA stability can account for the effect these sequences have on expression. Predictions of RNA structure reveal no obvious differences between the folding of TRP1 sequences modified to contain the stimulating TTAGATCTG motif compared with the non-stimulating TTAGTACTG derivative. Additional lines of evidence further support a DNA-based mechanism for IME $[21,40]$.

Several DNA-based mechanisms remain consistent with the results presented here and elsewhere, although some aspects remain puzzling for each. The ability of the TTNGATYTG motif to stimulate mRNA accumulation, while small changes to this sequence reduce or eliminate its effect on expression [32], suggests that there may be a protein such as a transcription factor that binds the motif in a sequence-specific 
manner. However, such a transcription factor would be unique for genes transcribed by RNA polymerase II in that it only functions when downstream of the transcription start site, and activates transcription several hundred nucleotides upstream of its binding site. The TTNGATYTG motif most closely resembles consensus sites for the GATA family of transcription factors [41,42], but GATA factor-binding sites do not meet the strict positional requirements characteristic of IME [43-45]. A second possible DNA-based model might include effects on local chromatin structure that favor transcript initiation, but this would not explain why these sequences must be downstream of the TSS to stimulate expression. A third possibility is that IME sequences influence the transcription machinery during elongation, elevating mRNA production by increasing processivity or the rate of transcription. This mechanism would not account for the ability of these sequences to boost expression in the absence of prior promoter activity [21].

Whatever the mechanism, it is clearly distinct from known effects of splicing, conventional transcription factor binding sites, and enhancer elements.

Implications in gene evolution and codon usage bias. Introns with high IMEter scores are associated with strongly expressed constitutive genes. It is possible that housekeeping genes have evolved sequences throughout their 5' ends that maximize ubiquitous expression. These sequences may be more commonly identified in introns due to the relative ease of generating and studying cDNA. Similar experiments exploring the effects of coding sequences and 5'-UTRs on expression are difficult to perform without introducing multiple confounding variables. Introns may also be more likely to contain stimulating sequences because they are under fewer evolutionary constraints than 5'-UTRs and coding sequences. However, the degenerate genetic code does allow for some flexibility in coding sequences. Not all codons are used with the same frequency, and this codon-usage bias can have dramatic effects on gene expression by diverse mechanisms (reviewed in[46]).

$\mathrm{N}$-terminal codon selection is thought to be especially important in determining expression levels. Effects on RNA secondary structure are the largest contributing factor, but still only explain about half of the variation observed $[47,48]$. In yeast, synonymous mutations at the 5' ends of genes have been shown to impact nucleosome positioning [49]. Synonymous substitutions also appear to occur less frequently at the 5' end of genes in mammalian populations (as determined by comparing evolution of the $B R C A-1$ gene in humans and dogs)[50]. In addition to codon usage, nucleotide frequency distributions also differ along the lengths of genes, suggesting that promoter proximal sequences may have evolved in response to pressures such as maximizing gene expression [51-53]. Further, optimizing expression by varying codon usage is more effective when adjacent codon pairs, rather than individual codons, are considered [54-56]. It is possible that some of the observed variation in expression associated with codon-usage bias is due to the inadvertent creation or destruction of stimulating sequences in coding regions.

Conclusion. What had been previously characterized as intron-mediated enhancement may not be limited to introns, and intron-mediated enhancement 
appears to be at least partially splicing independent. The ability of transcribed sequences near the start of genes to affect mRNA accumulation extends beyond introns and may include 5'-UTRs or coding sequences. These transcribed expression-stimulating sequences can be a useful addition to the promoters and enhancers used to regulate gene expression levels in transgenic or synthetic constructs.

\section{Materials and Methods}

Cloning of reporter gene fusions. The starting intronless TRP1:GUS template for all constructs included a $2.4 \mathrm{~Kb} T R P 1$ promoter fragment that extends from the middle of the upstream gene (At5g17980) through the first 8 amino acids of the third exon of TRP1 fused to the E. coli uidA (GUS) gene in the binary vector pEND4K [57]. To test the ability of the previously generated COR15a11L intron to stimulate expression from four additional locations (Figure 1), the intron, which is flanked by PstI sites [19], was cloned into previously generated TRP1:GUS constructs with PstI sites either 1136 or 1875 nucleotides downstream of the major transcription start site [20], or 21 or 324 nucleotides upstream of the TRP1 start codon [21].Transgenic plants in which the COR15a11L intron is located between the endogenous first and second exons of TRP1:GUS were previously described [32]. Other introns flanked by PstI sites are efficiently spliced at these locations [20,21,32].

To introduce the TTAGATCTG motif and TTAGTACTG control motif into exons (Figure 2), TRP1 sequences containing the described changes were synthesized by Biomatik (Wilmington, Deleware) and confirmed by sequencing. These fragments were used to replace analogous sequences between a PstI site engineered into the TRP1 5' UTR 87nt upstream of the start codon [21] and a BamHI site in the polylinker region connecting the TRPI and GUS coding sequences.

A 74 nucleotide BamHI fragment from the 3' end of the 5' UTR of $A 5 \operatorname{tg} 53000$ was used to replace an $80 \mathrm{nt} B a m H I$ to $B c l$ fragment of a modified version of the COR15a intron [29]. The inserted fragment from the At5g53000 5' UTR differs from the analogous endogenous fragment by three nucleotides. Changes were generated to introduce restriction sites via PCR mutagenesis:

Original: tțcaaaagacgatcctcttctcgaagaaactcgattcttgtggattcgatttcattaaggaattttgaattgtt Inserted: tçcaaaagacgatcctcttctcgaagaaactcgattcttgtggattcgatttcattaaggaattttgaattgga

The resulting hybrid intron was then cloned as a PstI fragment into a previously generated TRP1:GUS construct with a PStI site located between the endogenous first and second exon of TRP1 [20].

All fusions were then transformed into Agrobacterium tumefaciens by electroporation and introduced into Arabidopsis thaliana ecotype Columbia (Col) by floral dip as described [28]. 
Qualitative GUS expression assays. To compare the effect of the COR15a11L intron on TRP1:GUS expression from various locations (Figure 1B), five $\mathrm{T}_{2}$ seedlings from each of multiple lines whose segregation ratios indicated a single locus of transgene insertion were histochemically stained for GUS activity. To compare the enzyme activity of constructs containing the TTNGATYTG motif from within exons and introns (Figure $3 \mathrm{~B}$ ), $\mathrm{T}_{2}$ seedlings from 12 lines of unknown copy number for each construct and controls (intronless control: pAR281 [28], TRP1:GUS with COR15a6L intron: pAH3 [32]) were histochemically stained for GUS activity. In both cases, the plate of seedlings in buffer (10mM EDTA, $100 \mathrm{mM} \mathrm{NaP04} \mathrm{pH} 7.0$, 0.1\%Triton X-100) containing $0.5 \mathrm{mg} / \mathrm{mL} 5$-bromo-4-chloro-3-indolyl $B-D$ glucuronic acid (Calbiochem, La Jolla, CA, USA) was incubated at $37^{\circ}$ for one hour. The seedlings were washed in water and soaked in ethanol to remove chlorophyll.

Quantitative comparisons of enzyme activity and mRNA levels. Single-copy transgenic lines were identified for several key constructs, and mRNA levels on RNA gel blots and GUS activity in leaf extracts were measured as previously described [20]. In short, seeds from several dozen lines were screened for a 3:1 segregation ratio (kanamycin resistant: sensitive), and gel blots of DNA digested with restriction enzymes were probed with the GUS gene to determine transgene copy number. Single copy, homozygous lines were propagated to the $\mathrm{T}_{3}, \mathrm{~T}_{4}$, or $\mathrm{T}_{5}$ generation. RNA was extracted from 3-week-old seedlings, grown under constant light in Professional Growing Mix (Sun Gro Horticulture, Agawam, MA) at a density of 500 plants per $170 \mathrm{~cm}^{2}$ pot, using the Qiagen RNeasy kit. RNA gel blots were hybridized with a ${ }^{32} \mathrm{P}$-labeled GUS probe, and GUS mRNA levels in PhosphoImager scans were measured as pixels above background using Image Quant Software as described previously [29]. Quantitative measurements of GUS enzyme activity in leaf extracts were performed as described [20]. All mRNA and enzyme activity levels were normalized for total mRNA/protein and compared to the intronless control pAR281 [28].

Statistical differences in gene expression between constructs were analyzed by comparing Log mRNA levels using a mixed model that accounted for blot-to-blot differences, and adjusted for random effects per line and date of mRNA extraction for biological replicates. Residual normality was analyzed using a Wilk Shapiro test and homoscedasticity using a Levene ANOVA. Among the levels of categorical predictors, post hoc comparisons were based on least squares means using a protected least significant difference.

\section{References}

1. Butler JEF, Kadonaga JT. The RNA polymerase II core promoter: a key component in the regulation of gene expression. Genes Dev. 2002;16: 25832592. doi:10.1101/gad.1026202.The

2. Wray GA, Hahn MW, Abouheif E, Balhoff JP, Pizer M, Rockman M V., et al. The evolution of transcriptional regulation in eukaryotes. Molecular Biology and 
Evolution. 2003. pp. 1377-1419. doi:10.1093/molbev/msg140

3. Thomas MC, Chiang C-M. The general transcription machinery and general cofactors. Crit Rev Biochem Mol Biol. 2006;41: 105-178. doi:10.1080/10409230600648736

4. Danino YM, Even D, Ideses D, Juven-Gershon T. The core promoter: At the heart of gene expression. Biochimica et Biophysica Acta - Gene Regulatory Mechanisms. 2015. pp. 1116-1131. doi:10.1016/j.bbagrm.2015.04.003

5. Vernimmen D, Bickmore WA. The Hierarchy of Transcriptional Activation: From Enhancer to Promoter. Trends in Genetics. 2015. pp. 696-708. doi:10.1016/j.tig.2015.10.004

6. Pesole G, Mignone F, Gissi C, Grillo G, Licciulli F, Liuni S. Structural and functional features of eukaryotic mRNA untranslated regions. Gene. 2001. pp. 73-81. doi:10.1016/S0378-1119(01)00674-6

7. Hinnebusch AG, Ivanov IP, Sonenberg N. Translational control by 5 ? untranslated regions of eukaryotic mRNAs. Science (80- ). 2016;352: 14131416. doi:10.1126/science.aad9868

8. Wilkie GS, Dickson KS, Gray NK. Regulation of mRNA translation by 50-and 30]-UTR-binding factors. Trends Biochem Sci. 2003;28: 182-188. doi:10.1016/S0968-0004(03)00051-3

9. Stergachis AB, Haugen E, Shafer A, Fu W, Vernot B, Reynolds A, et al. Exonic Transcription Factor Binding Directs Codon Choice and Affects Protein Evolution. Science (80- ). 2013;342: 1367-1372. doi:10.1126/science. 1243490

10. Ahituv N. Exonic enhancers: proceed with caution in exome and genome sequencing studies. Genome Med. 2016;8: 14. doi:10.1186/s13073-016-02770

11. Kim MJ, Kim H, Shin JS, Chung CH, Ohlrogge JB, Suh MC. Seed-specific expression of sesame microsomal oleic acid desaturase is controlled by combinatorial properties between negative cis-regulatory elements in the SeFAD2 promoter and enhancers in the 50-UTR intron. Mol Genet Genomics. 2006;276: 351-368. doi:10.1007/s00438-006-0148-2

12. Deyholos MK. Separable Whorl-Specific Expression and Negative Regulation by Enhancer Elements within the AGAMOUS Second Intron. Plant Cell. 2000;12: 1799-1810. doi:10.1105/tpc.12.10.1799

13. Morello L, Bardini M, Sala F, Breviario D. A long leader intron of the Ostub16 rice \&\#x03B2;-tubulin gene is required for high-level gene expression and can autonomously promote transcription both in vivo and in vitro. Plant J. 2002;29: 33-44. doi:10.1046/j.0960-7412.2001.01192.x 
14. Wei CL, Wu Q, Vega VB, Chiu KP, Ng P, Zhang T, et al. A global map of p53 transcription-factor binding sites in the human genome. Cell. 2006;124: 207219. doi:10.1016/j.cell.2005.10.043

15. Dahan 0, Gingold H, Pilpel Y. Regulatory mechanisms and networks couple the different phases of gene expression. Trends in Genetics. 2011. pp. 316-322. doi:10.1016/j.tig.2011.05.008

16. Le Hir H, Gatfield D, Izaurralde E, Moore MJ. The exon-exon junction complex provides a binding platform for factors involved in mRNA export and nonsense-mediated mRNA decay. EMBO J. 2001;20: 4987-4997. doi:10.1093/emboj/20.17.4987

17. Wiegand HL, Lu S, Cullen BR. Exon junction complexes mediate the enhancing effect of splicing on mRNA expression. Proc Natl Acad Sci U S A. 2003;100: 11327-32. doi:10.1073/pnas.1934877100

18. Koga M, Hayashi M, Kaida D. Splicing inhibition decreases phosphorylation level of Ser2 in Pol II CTD. Nucleic Acids Res. 2015;43: 8258-8267. doi:10.1093/nar/gkv740

19. Gallegos JE, Rose AB. The enduring mystery of intron-mediated enhancement. Plant Science. Elsevier Ireland Ltd; 2015. pp. 8-15.

20. Rose AB. The effect of intron location on intron-mediated enhancement of gene expression in Arabidopsis. Plant J. 2004;40: 744-751. doi:10.1111/j.1365-313X.2004.02247.x

21. Gallegos JE, Rose AB. Intron DNA Sequences Can Be More Important Than the Proximal Promoter in Determining the Site of Transcript Initiation. Plant Cell . 2017; doi:10.1105/tpc.17.00020

22. Callis J, Fromm M, Walbot V. Introns increase gene expression in cultured maize cells. Genes Dev. 1987;1: 1183-1200. doi:10.1101/gad.1.10.1183

23. Jeong Y-M. Distinct Roles of the First Introns on the Expression of Arabidopsis Profilin Gene Family Members. Plant Physiol. 2006;140: 196-209. doi:10.1104/pp.105.071316

24. Snowden KC, Buchholz WG, Hall TC. Intron position affects expression from the tpi promoter in rice. Plant Mol Biol. 1996;31: 689-692.

25. Rose AB. Requirements for intron-mediated enhancement of gene expression in Arabidopsis. Rna. 2002;8: 1444-1453. doi:10.1017/S1355838202020551

26. Clancy M, Hannah LC. Splicing of the maize Sh1 first intron is essential for enhancement of gene expression, and a T-rich motif increases expression without affecting splicing. Plant Physiol. 2002;130: 918-29. doi:10.1104/pp.008235 
27. Akua T, Berezin I, Shaul 0. The leader intron of AtMHX can elicit, in the absence of splicing, low-level intron-mediated enhancement that depends on the internal intron sequence. BMC Plant Biol. 2010;10: 93. doi:10.1186/14712229-10-93

28. Rose AB, Beliakoff JA. Intron-mediated enhancement of gene expression independent of unique intron sequences and splicing. Plant Physiol. 2000;122: 535-542. doi:10.1104/pp.122.2.535

29. Rose AB, Elfersi T, Parra G, Korf I. Promoter-proximal introns in Arabidopsis thaliana are enriched in dispersed signals that elevate gene expression. Plant Cell. 2008;20: 543-551. doi:10.1105/tpc.107.057190

30. Zhang N, McHale LK, Finer JJ. Isolation and characterization of "GmScream" promoters that regulate highly expressing soybean (Glycine max Merr.) genes. Plant Sci. 2015;241: 189-198. doi:10.1016/j.plantsci.2015.10.010

31. Aguilar-Hernández V, Guzmán P. Spliceosomal introns in the 5' untranslated region of plant BTL RING-H2 ubiquitin ligases are evolutionary conserved and required for gene expression. BMC Plant Biol. 2013;13: 179. doi:10.1186/1471-2229-13-179

32. Rose AB, Carter A, Korf I, Kojima N. Intron sequences that stimulate gene expression in Arabidopsis. Plant Mol Biol. 2016;92: 337-346. doi:10.1007/s11103-016-0516-1

33. Rose AB, Casselman AL, Last RL. A Phosphoribosylanthranilate Transferase Gene Is Defective in Blue Fluorescent Arabidopsis thaliana Tryptophan Mutants. Plant Physiol. 1992;100: 582-592. doi:10.1104/pp.100.2.582

34. von HEIJNE G, STEPPUHN J, HERRMANN RG. Domain structure of mitochondrial and chloroplast targeting peptides. Eur J Biochem. 1989;180: 535-545. doi:10.1111/j.1432-1033.1989.tb14679.x

35. Inoue K, Glaser E. Processing and Degradation of Chloroplast Extension Peptides. In: Theg SM, Wollman F-A, editors. Plastid Biology. New York, NY: Springer New York; 2014. pp. 305-323. doi:10.1007/978-1-4939-1136-3_12

36. Parra G, Bradnam K, Rose AB, Korf I. Comparative and functional analysis of intron-mediated enhancement signals reveals conserved features among plants. Nucleic Acids Res. 2011;39: 5328-5337. doi:10.1093/nar/gkr043

37. Ahn CS, Ahn H-K, Pai H-S. Overexpression of the PP2A regulatory subunit Tap46 leads to enhanced plant growth through stimulation of the TOR signalling pathway. J Exp Bot. 2015;66: 827-840. Available: http://dx.doi.org/10.1093/jxb/eru438

38. Moabbi a. M, Agarwal N, El Kaderi B, Ansari a. Role for gene looping in intron-mediated enhancement of transcription. Proc Natl Acad Sci. 2012;109: 
8505-8510. doi:10.1073/pnas.1112400109

39. Agarwal N, Ansari A. Enhancement of Transcription by a Splicing-Competent Intron Is Dependent on Promoter Directionality. PLoS Genet. 2016;12. doi:10.1371/journal.pgen.1006047

40. Rose AB, Emami S, Bradnam K, Korf I. Evidence for a DNA-Based Mechanism of Intron-Mediated Enhancement. Front Plant Sci. 2011;2: 1-9. doi:10.3389/fpls.2011.00098

41. O'Malley RC, Huang S shan C, Song L, Lewsey MG, Bartlett A, Nery JR, et al. Erratum: Cistrome and Epicistrome Features Shape the Regulatory DNA Landscape (Cell (2016) 165(5) (1280???1292)). Cell. 2016. p. 1598. doi:10.1016/j.cell.2016.08.063

42. Weirauch MT, Yang A, Albu M, Cote AG, Montenegro-Montero A, Drewe P, et al. Determination and Inference of Eukaryotic Transcription Factor Sequence Specificity. Cell. 2014;158: 1431-1443. doi:10.1016/j.cell.2014.08.009

43. Sugimoto K, Takeda S, Hirochika H. Transcriptional activation mediated by binding of a plant GATA-type zinc finger protein AGP1 to the AG-motif (AGATCCAA) of the wound-inducible Myb gene NtMyb2. Plant J. 2003;36: 550-564. doi:10.1046/j.1365-313X.2003.01899.x

44. Jeong MJ, Shih MC. Interaction of a GATA factor with cis-acting elements involved in light regulation of nuclear genes encoding chloroplast glyceraldehyde-3-phosphate dehydrogenase in Arabidopsis. Biochem Biophys Res Commun. 2003;300: 555-562. doi:10.1016/S0006-291X(02)02892-9

45. Endo H, Yamaguchi M, Tamura T, Nakano Y, Nishikubo N, Yoneda A, et al. Multiple classes of transcription factors regulate the expression of VASCULAR-RELATED NAC-DOMAIN7, a master switch of xylem vessel differentiation. Plant Cell Physiol. 2015;56: 242-254. doi:10.1093/pcp/pcu134

46. Plotkin JB, Kudla G. Synonymous but not the same: the causes and consequences of codon bias. Nat Rev Genet. 2011;12: 32-42. doi:10.1038/nrg2899

47. Kudla G, Murray AW, Tollervey D, Plotkin JB. Coding-sequence determinants of gene expression in Escherichia coli. Science. 2009;324: 255-8. doi:10.1126/science. 1170160

48. Goodman DB, Church GM, Kosuri S. Causes and Effects of N-Terminal Codon Bias in Bacterial Genes. Science (80- ). 2013;342: 475-479. doi:10.1126/science.1241934

49. Warnecke T, Batada NN, Hurst LD. The impact of the nucleosome code on protein-coding sequence evolution in yeast. PLoS Genet. 2008;4. 
doi:10.1371/journal.pgen.1000250

50. Chamary J V, Parmley JL, Hurst LD. Hearing silence: non-neutral evolution at synonymous sites in mammals. Nat Rev Genet. 2006;7: 98-108. doi:10.1038/nrg1770

51. Louie E, Ott J, Majewski J. Nucleotide frequency variation across human genes. Genome Res. 2003;13: 2594-2601. doi:10.1101/gr.1317703

52. Serres-Giardi L, Belkhir K, David J, Glemin S. Patterns and evolution of nucleotide landscapes in seed plants. Plant Celll. 2012;24: 1379-1397. doi:10.1105/tpc.111.093674

53. Stoletzki $N$. The surprising negative correlation of gene length and optimal codon use--disentangling translational selection from GC-biased gene conversion in yeast. BMC Evol Biol. 2011;11: 93. doi:10.1186/1471-2148-1193

54. Lanza AM, Curran K a, Rey LG, Alper HS. A condition-specific codon optimization approach for improved heterologous gene expression in Saccharomyces cerevisiae. BMC Syst Biol. 2014;8: 33. doi:10.1186/17520509-8-33

55. Chung BKS, Yusufi FNK, Mariati, Yang Y, Lee DY. Enhanced expression of codon optimized interferon gamma in CHO cells. J Biotechnol. 2013;167: 326333. doi:10.1016/j.jbiotec.2013.07.011

56. Ahn J, Jang MJ, Ang KS, Lee H, Choi ES, Lee DY. Codon optimization of Saccharomyces cerevisiae mating factor alpha prepro-leader to improve recombinant protein production in Pichia pastoris. Biotechnol Lett. 2016;38: 2137-2143. doi:10.1007/s10529-016-2203-3

57. Rose $A B$, Last RL. Introns act post-transcriptionally to increase expression of the Arabidopsis thaliana tryptophan pathway gene PAT1. Plant J. Blackwell Science Ltd; 1997;11: 455-464. doi:10.1046/j.1365-313X.1997.11030455.x 

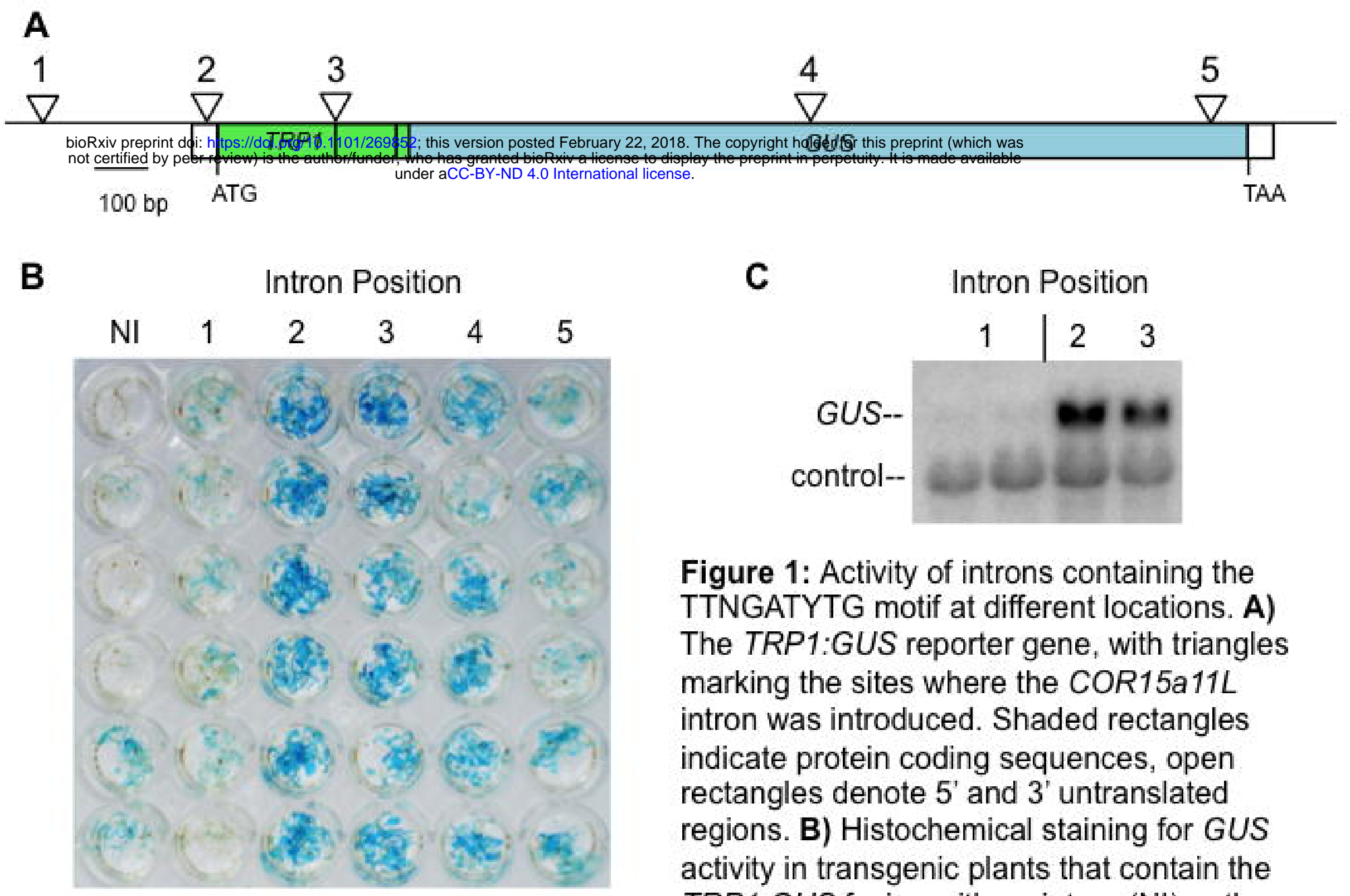

Figure 1: Activity of introns containing the TTNGATYTG motif at different locations. A) The TRP1:GUS reporter gene, with triangles marking the sites where the COR15a11L intron was introduced. Shaded rectangles indicate protein coding sequences, open rectangles denote $5^{\prime}$ and $3^{\prime}$ untranslated regions. B) Histochemical staining for GUS activity in transgenic plants that contain the TRP1:GUS fusion with no intron (NI) or the COR15a11 L intron at the position indicated at the top of each column. Each well contains five $T_{2}$ seedlings from an independent transgenic line. C) RNA gel blot probed with GUS and a loading control (the endogenous TRP1 gene). Each lane contains RNA from an independent single-copy homozygous line. 
A)

$\stackrel{\text { Pstl }}{\text { ctgcagtattagagaggaaggaacgaaccatcatagaaatctctccttcgttcaagtttttctctactgt }}$ gttggttgagcaatcgagtgATGGTTATTGCGGTGGCGACGACGAGCTCCATTGTTTCCGGAATCAAGCT TTCAGGGATCTTAACT,TCGTTCAAC,GCC,GTA, GGAGTTAGATTTTTCCGACATTGATTCGGCGACGATTCTCATCGATCGGAGCT,GTTTCCCCAATTCGTG $\nabla$

GGGATGCTCAATCTTCCTTTAGTCGCAGCTCTTTCGCTTGCTCTCAGAATCTCGGTTTGAGCGTGGATT

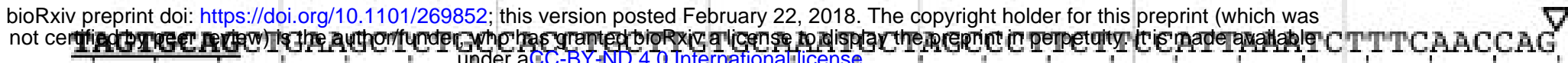

TTGATTGAAACCTTGATTGATCGGtctagaggatcc $\underbrace{\text { BamHI }}_{X \text { bal }}$

B) MVIAVATTSSIVSGIKLSGILTSFNAVDDASSSCGRSNLTGVRIFPTLSRRRFSSIGAVSPIRGDAQ SSFSRSSFACSQNLGLSGGFSAAEALPPACANASPSSIKSFNQLIETLIDR

C)

\begin{tabular}{|c|c|c|c|}
\hline \multirow{3}{*}{$\underset{\text { in }}{\stackrel{\alpha}{5}}$} & Wild type & TTAGATCTG & TTAGTACTG \\
\hline & gttcaagtt & ttagatctg & ttagtactg \\
\hline & tactgtgtt & ttagatctg & ttagtactg \\
\hline \multirow{8}{*}{ 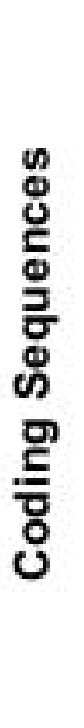 } & AG CTT TCA G & TTAGA TCT G & TTAGT ACT G \\
\hline & $\begin{array}{llll}K & L & S & G\end{array}$ & I $R \quad S \quad G$ & $\begin{array}{llll} & \mathbf{S} & \mathbf{T} & \mathbf{G}\end{array}$ \\
\hline & TT TTT CCG A & TTAGA TCT G & TT AGT ACT G \\
\hline & $\begin{array}{llll}\text { I } & F & P & T\end{array}$ & I $\quad R \quad S \quad A$ & $\begin{array}{lll}\mathrm{I} & \mathrm{S} & \mathrm{T}\end{array}$ \\
\hline & GA CGA TTC T & TTAGA TCT G & TTAGT ACT G \\
\hline & $\begin{array}{llll}R & R & F & S\end{array}$ & L $R \quad S \quad A$ & $\begin{array}{llll}\mathbf{L} & \mathrm{S} & \mathrm{T} & \mathrm{A}\end{array}$ \\
\hline & TT AGT GCA G & TT AGA TCT G & TT AGT ACT G \\
\hline & $\begin{array}{llll}F & S & A & A\end{array}$ & $\begin{array}{llll}F & R & S & A\end{array}$ & $\begin{array}{llll}F & S & T & A\end{array}$ \\
\hline
\end{tabular}

Figure 2: Details of changes to TRPI:GUS sequence to introduce motifs. A) Uppercase letters mark TRPI coding sequences with the start codon highlighted. Lowercase letters indicate the TRPI 5' UTR and the sites used to fuse TRP1 to GUS. The inverted triangles show the location of introns in the endogenous TRP1 gene. Sequences that are underlined and bold were changed to either TTAGATCTG or TTAGIACTG. B) The amino acids encoded by the TRPI sequence shown in $\mathrm{A}$, with the regions affected by introducing the motifs underlined and in bold. C) Details of the nucleotides and amino acids changed. 


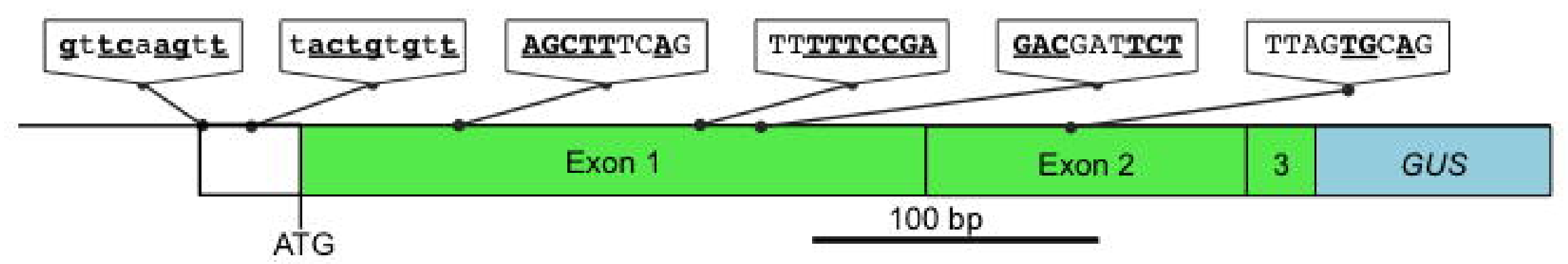

bioRxiv preprint doi: https://doi.org/10.1101/269852; this version posted February 22, 2018. The copyright holder for this preprint (which was not certified by peer review) is the author/funder, who has granted bioRxiv a license to display the preprint in perpetuity. It is made available under aCC-BY-ND 4.0 International license.

No Intron

B
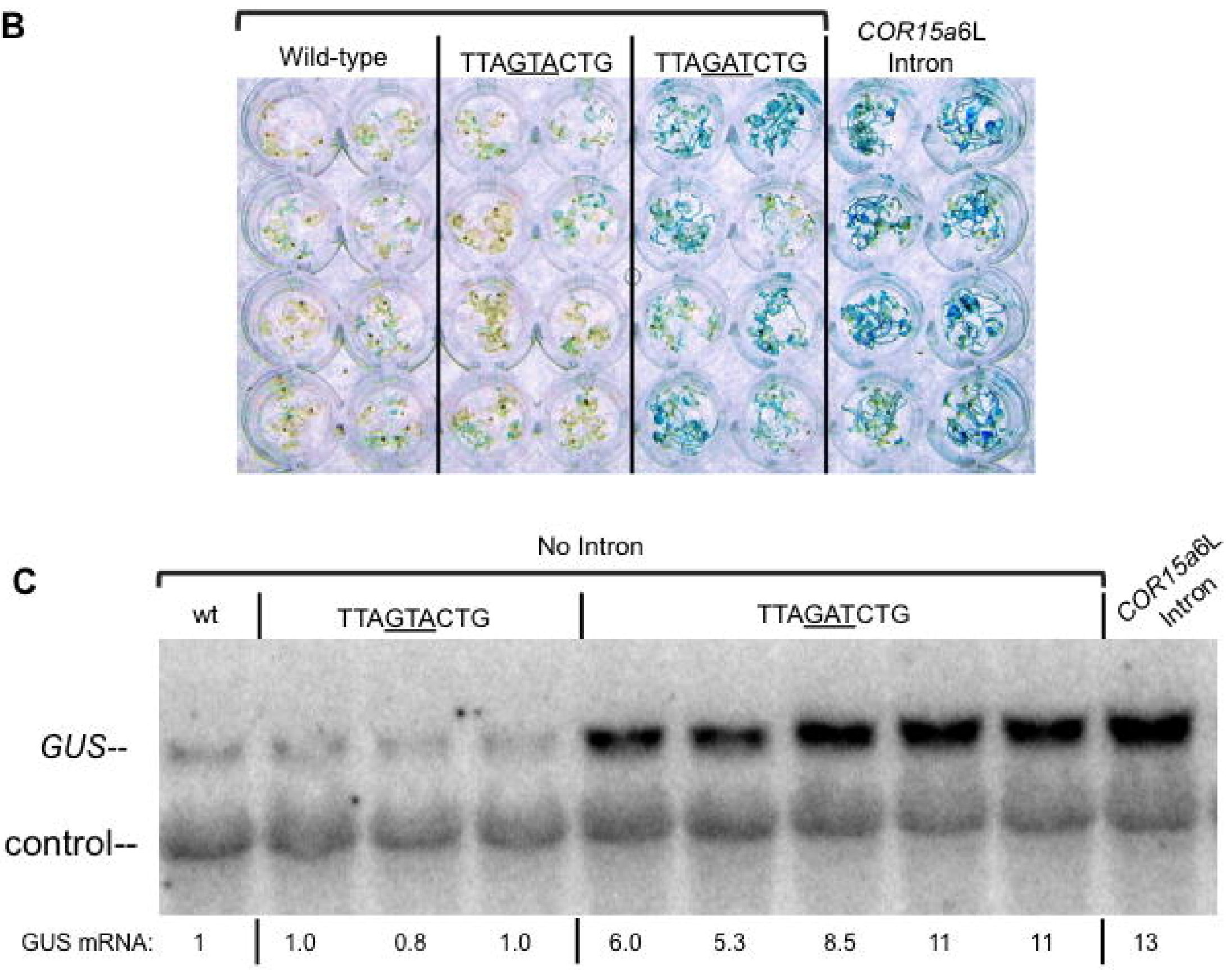

Figure 3: Testing the ability of the TTNGATYTG motif to stimulate expression from within exons. A) The nucleotides in the sequences at the indicated locations in a TRP1:GUS fusion that were changed to match the motif are bold and underlined. The designations of exons 1,2, and 3 refer to the location of the same sequences in the endogenous TRP1 gene. The COR15a6L intron in the control construct is located at the junction between TRP1 exon 1 and 2 sequences. No other constructs contain an intron. B) Histochemical staining for GUS activity in transgenic plants that contain the indicated TRP1:GUS fusions. Each well contains five $T_{2}$ seedlings from an independent line of unknown copy number. C) RNA gel blot probed with GUS and a loading control (the endogenous TRP1 gene). Each adjacent lane with the same label represents an independent single-copy homozygous line. 
Figure 4: 5'-UTR sequences can stimulate expression from within an intron. RNA gel blot of RNA from transgenic lines containing a TRP1:GUS fusion with no intron, the COR15a intron, or a

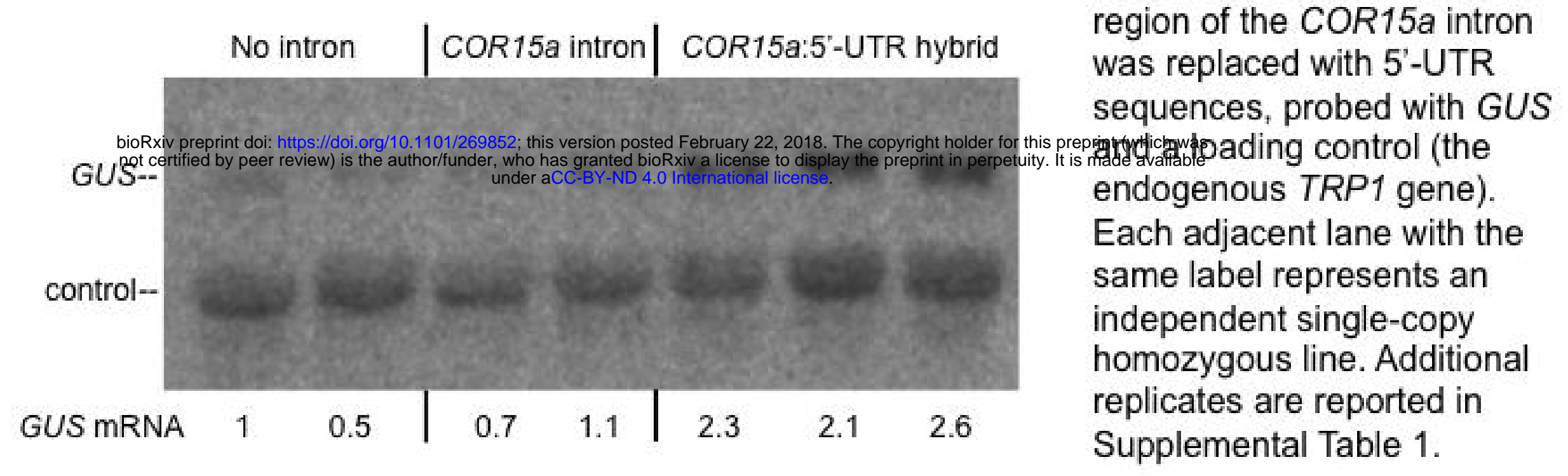

